Disponível em:

http://editora.unoesc.edu.br/index.php/race

RACE, Joaçaba, v. 16, n. 2, p. 573-602, maio/ago. 2017

\title{
IMPACTO DOS RATINGS DE CRÉDITO NAS AÇÕES DE EMPRESAS DE CAPITAL ABERTO NO BRASIL
}

The impact of rating changes in Brazilian financial markets

\section{Rafaela Augusta Cunha Silveira}

E-mail: rafaelaacsilveira@hotmail.com

Bacharel em Controladoria e Finanças pela Universidade Federal de Minas Gerais; Graduanda em Ciências Contábeis pelo Instituto Brasileiro de Mercado de Capitais.

\begin{abstract}
Renata Turola Takamatsu
E-mail: rettakamatsu@gmail.com

Doutora em Controladoria e Contabilidade pela Universidade de São Paulo; Mestre em Controladoria e Contabilidade pela Universidade de São Paulo; Professora Adjunta no Departamento de Ciências Contábeis da Universidade Federal de Minas Gerais.

Bruna Camargos Avelino

E-mail: bcavelino@gmail.com

Doutora em Controladoria e Contabilidade pela Universidade de São Paulo;

Mestre em Ciências Contábeis pela Universidade Federal de Minas Gerais;

Professora Adjunta no Departamento de Ciências Contábeis da Universidade Federal de Minas Gerais. Endereço para contato: Avenida Antonio Carlos, 6627, Pampulha, 31270-901, Belo Horizonte, Minas Gerais, Brasil.
\end{abstract}

Artigo recebido em 08 de junho de 2016. Aceito em 28 de novembro de 2016. 


\section{Resumo}

O rating de crédito expressa uma opinião, por intermédio de escalas, sobre a qualidade do crédito de empresas, utilizado-a como medida de avaliação de risco no mercado. Agências de classificação de risco de crédito, como a Moody’s, divulgam os ratings que atribuem às empresas. Primeiramente, essas agências emitem o new rating, que representa o primeiro rating da companhia, e, posteriormente, essa emissão pode apresentar variações, denominadas upgrades e downgrades, relativas a boas e más notícias, respectivamente. Além disso, os ratings podem ser colocados em uma Watchlist quando, em breve, pode haver uma mudança do rating para downgrade ou para upgrade. O objetivo com este estudo consistiu, diante do que foi tratado, em abordar o impacto do rating de crédito sobre os preços das ações de empresas listadas na bolsa de valores brasileira. Para alcançar o objetivo proposto, foi analisada uma amostra de 44 empresas comercializadas na BM\&FBovespa e 65 ratings nacionais de longo prazo emitidos pela Moody’s entre 2000 e 2015. Utilizou-se a metodologia de estudo de eventos, com os retornos normais calculados pelo modelo de retornos ajustados ao risco e ao mercado, e o Teste-F e o Teste-T para verificar a significância dos resultados. As análises finais evidenciaram que os preços das ações não são afetados de forma significativa pelas divulgações dos new ratings, downgrades, upgrades, on watch - possible downgrades e on watch - possible upgrades em nenhuma janela do evento, indicando que os ratings, para a amostra analisada, não trazem novas informações ao mercado.

Palavras-chave: Ações. Rating. Estudo de eventos. Retornos anormais.

\section{Abstract}

Credit ratings are used as a mean to investors get new information on the companies by reducing the information asymmetry in the market. Thus, the rating is an important mean of business information with investors, enabling share prices relating to companies react to it. Branches of credit rating as Moody's, disclose the ratings they assign to companies. First, the agency issues the new rating, which represents the company's first rating, then this issue may vary, upgrades and downgrades calls relating to good and bad news respectively. In addition, the ratings could be placed in a Watchlist when, soon there may be a change to the rating downgrade or upgrade. The purpose of this study was to discuss the impact that the credit rating has on stock prices of companies listed on the Brazilian stock exchange. For a sample of 44 companies traded on BM\&FBovespa and 65 long-term national ratings issued by Moody's between 2000 and 2015, we used the event study methodology, with normal returns calculated by the model of returns adjusted for risk and market the F-Test and T-Test to test the significance of the results. The final analysis showed that stock prices are not significantly affected by the disclosures of new ratings, downgrades, upgrades, on watch - possible downgrades and on watch - possible upgrades in any event window, indicating that the ratings do not bring new information to the market.

Keywords: Stocks. Rating. Event studies. Abnormal returns. 


\section{INTRODUÇÃO}

O rating de crédito expressa uma opinião, por intermédio de escalas, sobre a qualidade do crédito de empresas, que é utilizado como uma medida de avaliação de risco no mercado. A Moody’s (2015) afirma que os ratings antecipam ou apontam o potencial de perda de crédito das companhias em razão de três motivos relacionados ao pagamento: a não realização, o atraso dele e o pagamento parcial ao investidor.

Os ratings são de suma importância para os investidores medirem o risco de seus investimentos. De acordo com a Moody's (2015), os empreendedores os utilizam como um auxílio no momento de precificar o risco de crédito em títulos de renda fixa que eles estejam dispostos a comprar ou vender. Muitos deles também lidam com o rating como um limite em seus critérios de investimento. Além disso, essa medida de avaliação de risco serve como uma forma de aumentar os horizontes para mercados ou outros títulos aos quais quem investe não atende com a sua própria análise.

Segundo Damasceno, Artes e Minardi (2008), os sistemas de rating de crédito estão ficando cada vez mais importantes, principalmente em razão das regras existentes. De acordo com esse autor, o Acordo de Basileia II autoriza os bancos a utilizarem ratings externos de agências, bem como a elaborarem sistemas internos que sejam capazes de administrar o risco de crédito da sua carteira de empréstimos e títulos de dívida.

Esses ratings de crédito são publicados por agências de classificação de risco, como a Fitch Ratings, a Moody’s e a Standard\&Poor, consideradas as principais no mercado. Essas agências utilizam parâmetros para os ratings que se apoiam especialmente na capacidade de criação de caixa da entidade emissora e nas garantias e fianças por ela disponibilizadas (BM\&FBOVESPA, 2015).

Papaikonomou (2010) ressalta que as agências de rating de crédito proporcionam um serviço de valor substancial para o mercado de capitais se elas entregam uma avaliação de confiança e que não esteja sujeita à probabilidade relativa de default de instrumentos financeiros de crédito, o que possibilita que a assimetria de informação seja diminuída entre os investidores e as companhias que emitem o título.

Murcia (2013) afirma que as agências são um meio importante para mediar os emissores e os investidores, em decorrência da colaboração que elas têm para o bom andamento do mercado financeiro conforme ajudam na redução da assimetria informacional e da seleção adversa. Desse modo, ainda segundo Murcia (2013), o investidor consegue tomar melhores decisões econômicas apoiando-se no rating emitido pelas agências, visto que este será capaz de distinguir os títulos que têm boa qualidade 
dos de pior qualidade, o que, consequentemente, resultaria em uma melhor alocação de recursos no mercado.

Salienta-se, no entanto, que a qualquer momento os ratings de crédito podem sofrer alterações, conhecidas como upgrades e downgrades. Upgrades e downgrades são, respectivamente, um aumento e um rebaixamento no rating de crédito da empresa. Geralmente, essas alterações acontecem por causa da ocorrência de boas e más notícias sobre as companhias.

Além de sofrerem alterações, os ratings podem passar por uma revisão, sendo colocados em Watchlist ou Credit Watch (revisão de rating). Segundo a Moody's (2015), isso ocorre quando ele está em reavaliação para uma provável ascensão (on watch - possible upgrade) ou rebaixamento (on watch - possible downgrade) do rating, ou seja, quando a agência de classificação de risco de crédito considera que uma alteração no rating de crédito poderá ocorrer no curto prazo (HILL, 2004).

Tendo conhecimento dessas alterações que os ratings de crédito das empresas podem sofrer no decorrer do tempo, o problema que se visa responder neste estudo é: qual a relação entre os anúncios de ratings iniciais, de downgrades, de upgrades e de Watchlist e os retornos das ações de companhias de capital aberto do mercado brasileiro? O objetivo com esta pesquisa consistiu, portanto, em descobrir qual é o efeito dos anúncios de ratings iniciais, da Watchlist e das variações dos ratings de crédito (downgrades e upgrades) de companhias abertas do mercado brasileiro, obtidos por meio da Moody’s, nos retornos das ações dessas empresas.

O estudo se justifica pela relevância da temática em análise, além da falta de consenso evidenciada pela literatura em relação ao conteúdo das informações do rating de crédito. Uma parcela significativa das pesquisas encontradas sobre o tema tem sido realizada no âmbito internacional, geralmente em países de economias desenvolvidas. Pretende-se, portanto, preencher tal lacuna encontrada no Brasil, priorizando o estudo em uma economia emergente.

\section{REVISÃO TEÓRICA}

\subsection{TEORIA DA EFICIÊNCIA DE MERCADO}

A Teoria da Eficiência de Mercado é retratada em uma série de estudos financeiros que nela baseiam seus resultados. Um mercado eficiente, de acordo com Fama (1970), é aquele em que os preços sempre refletirão todas as informações disponíveis 
no mercado, sendo estas acessíveis a todos os participantes. Nesse mercado, não é possível obter lucros econômicos por negociações utilizando como base as informações disponíveis (JENSEN, 1978).

Segundo Maluf Filho (1991), a eficiência de mercado pode ser classificada como fraca, semiforte e forte, que se diferenciarão pelo tipo de informação disponível. A classificação chamada de fraca refere-se à situação em que o preço atual está englobando todas as informações incluídas nas séries anteriores de preços. O nível semiforte implica, assim como o anterior, o comportamento histórico dos preços, porém, também trará a adaptação dos preços aos conteúdos que foram tornados públicos, como demonstrativos financeiros publicados e anúncios de distribuição de proventos. Por fim, na forma forte, o preço refletirá as informações importantes que até então não foram divulgadas ao público (privilegiadas). Ainda segundo Maluf Filho (1991), essas três classificações têm uma regra de dominância, ou seja, o nível semiforte somente é atingido se as condições do nível fraco forem realizadas. Isso acontece com o nível forte, que precisa que os requisitos da forma fraca e semiforte sejam alcançados.

Estudar anúncios de rating está diretamente ligado ao estudo da eficiência de mercado. De acordo com Murcia (2013), o nível de eficiência em que o mercado de ações incorpora, processa e reflete uma nova informação nos valores das ações causa amplas conclusões a respeito dos seus sistemas internos. Assim, se esse mercado é forte no que diz respeito ao nível de eficiência, mesmo que as agências de classificação façam uso de informações privilegiadas, o mercado não precisaria responder de maneira anormal no valor das ações quando há a divulgação do rating. Porém, segundo Taib et al. (2009), caso os anúncios de revisão de rating publicados pelas agências de crédito originarem retornos anormais, esse fato poderá indicar o nível semiforte da hipótese do mercado eficiente ou a interferência da informação privilegiada, que apenas é acessível às agências de classificação.

\subsection{TEORIA DA ASSIMETRIA INFORMACIONAL}

Belo e Brasil (2006) afirmam que o conceito de assimetria informacional está relacionado a um evento no qual alguns agentes econômicos são dotados de mais informações do que os demais. Portando, esses agentes possuem algumas informações privilegiadas que os auxiliam a conseguirem melhores resultados, estando em uma posição vantajosa frente aos outros. Além disso, quando há assimetria informacional no mercado, provavelmente haverá conflitos de interesse. 
De acordo com Abad-Romero e Robles-Fernandez (2006), as agências de classificação de crédito são compostas por informações importantes e privadas sobre certa empresa; desse modo, o rating pode proporcionar informação complementar ao mercado financeiro sobre o valor total da companhia. Logo, é possível reconhecer que o rating auxilia na diminuição da assimetria informacional em relação à empresa emissora e aos investidores (LI; VISALTANACHOTI; KESAYAN, 2003).

\subsection{RISCO DE CRÉDITO}

Segundo Sih (2006), medir o risco de uma empresa está relacionado à contribuição que isso terá nas tomadas de decisões em relação a prováveis investimentos nela, visto que esses investimentos estão se tornando cada vez mais globais e diversos. Definir em quais companhias aplicar dinheiro é um desafio, assim, o investidor deverá estar ciente dos riscos que ele correrá. Um desses riscos é o risco de crédito, foco principal nesta pesquisa.

Para Sales (2006), a possibilidade de um emissor se tornar inadimplente, ou seja, de não pagar os juros ou principal é conhecida como risco de crédito. A respeito disso, Callado et al. (2008) afirmam que a partir do final de década de 1980, a busca por informações associadas à avaliação desse risco começou a se ampliar no mercado financeiro internacional. Desse modo, foram criados diversos métodos para a avaliação desse risco, que disponibilizam classificações de risco de crédito aos investidores. Ainda segundo Callado et al. (2008), essas classificações representam o nível de risco exclusivo de certa companhia, por meio da probabilidade de default, ou seja, são os ratings de crédito publicados pelas agências classificadoras.

\subsection{AS AGÊNCIAS DE RATING}

A globalização dos mercados financeiros, a elaboração de novos produtos e a estabilidade econômica de lugares ainda pouco notórios pelos investidores internacionais foram essenciais no auxílio do crescimento das agências de rating, assim como para maior sofisticação dos parâmetros e dos métodos utilizados para as avaliações de crédito. Como consequência, atualmente existem amplas informações concedidas por essas agências (DAMASCENO; ARTES; MINARDI, 2008).

Segundo Murcia (2013), as agências de classificação emitem ratings para companhias (ratings corporativos), títulos de dívida, países (ratings soberanos) e ci- 
dades. Além disso, o autor destaca que os ratings podem ser diferentes em razão da moeda da emissão, referirem a vários títulos de crédito, como títulos públicos, debêntures e notas promissórias e serem de longo ou curto prazo.

Os ratings de longo prazo referem-se a emissores ou obrigações com expiração real de um ano ou superior a um e mostram a possibilidade de um default em pagamentos contratualmente prometidos e o prejuízo financeiro esperado caso ocorra default. Já os de curto prazo dizem respeito a obrigações com expiração real de 13 meses ou menos e manifestam a possibilidade de um default nos pagamentos contratualmente prometidos (MOODY'S, 2015).

O presente estudo utilizou os ratings corporativos, emitidos pela Moody’s, de longo prazo e em escala nacional, definidos como:

[...] avaliações relativas da qualidade de crédito dentro de um restrito grupo de pares, quando comparado com o universo total das entidades classificadas pela Moody's, e são, portanto, diferentes dos ratings em escala global da Moody’s. Os Ratings na Escala Nacional podem ser atribuídos a qualquer obrigação de dívida oferecida dentro de um dado mercado doméstico de capitais, denominada ou não na moeda local relevante. (MOODY'S, 2015, p. 1).

A Moody’s (2015) afirma que os seus ratings corporativos são ratings de longo prazo que refletem a possibilidade de um conjunto corporativo pagar suas dívidas e do provável prejuízo financeiro na ocorrência de default. Conforme Sih (2006), o rating corporativo é fundamentado em dados atualizados que são concedidos pelas empresas ou por outras fontes julgadas seguras. Este é revisado periodicamente, e tais revisões podem causar mudanças, que refletirão a opinião da agência de que houve uma melhora ou uma piora na solvência de crédito da companhia (ABAD-ROMERO; ROBLES-FERNANDEZ, 2006). Isso quer dizer que, caso haja uma melhora na solvência de crédito da empresa, ocorrerá um upgrade e, caso contrário, um downgrade.

Segundo Murcia (2013), o new rating (o primeiro rating emitido) de uma companhia ou de uma emissão e, também, as suas revisões, podem ser distintos, dependendo da agência que o atribui, ou seja, é possível que uma única empresa ou emissão possa ter avalições diferentes, já que existem várias agências de classificação de crédito.

Quando a probabilidade de alteração do rating é alta, a empresa é colocada na Watchlist e retirada quando acontece um downgrade ou um upgrade ou quando o rating é atualizado. Porém, a entidade pode ter a divulgação de alteração de rating depois que a empresa foi incluída na Watchlist, ou esse anúncio pode acontecer como 
uma revisão direta, isto é, quando a companhia não estava listada na Watchlist (MURCIA, 2013).

\subsection{ESTUDOS ANTERIORES}

O impacto do rating de crédito nas ações de empresas é um tema estudado por autores distintos, sendo um assunto que apresenta resultados contraditórios. Isso acontece pois algumas pesquisas encontraram resultados iguais para os downgrades e os upgrades, e outras acharam resultados divergentes para eles.

Segundo Calderoni, Colla e Gatti (2009), a maioria dos estudos existentes concorda com o resultado de que as consequências da alteração de rating de curto prazo sobre os preços das ações são assimétricas: downgrades produzem retornos anormais negativos significativos e em relação aos upgrades não há nenhum impacto significativo, positivo ou negativo, ou seja, os resultados empíricos contradizem a teoria do mercado eficiente.

Abad-Romero e Robles-Fernandez (2006), utilizando ratings emitidos pela Moody’s e Standard\&Poor e Fitch IBCA, observaram o impacto das alterações dos ratings dos títulos das companhias nos retornos de mercado e no risco sistemático, ou seja, no risco do mercado de entidades comercializadas no mercado de ações da Espanha, de janeiro de 1990 a fevereiro de 2003. Como resultado, não encontraram retornos anormais significativos para downgrades, mas acharam retornos anormais negativos significativos para upgrades, justificados por razões internas das entidades.

Creighton, Gower e Richards (2007), que desenvolveram um estudo sobre os efeitos das mudanças de ratings nos preços de títulos de dívida e de ações na Austrália, concluíram que os preços das ações tendem a cair em dias de downgrades e a aumentar em dias de upgrades, isto é, os resultados desses autores coincidiram com a teoria do mercado eficiente. Eles também afirmaram que suas estimativas sobre os efeitos dos preços em relação aos anúncios de rating são pequenas, o que sugere que as agências não são normalmente vistas como detentoras de informações significativas que já não estão no domínio público.

Callado et al. (2008) realizaram um estudo em que foi observado o efeito da divulgação do new rating de instituições financeiras negociadas na BM\&FBovespa, utilizando um estudo de eventos. Os autores não encontraram indícios significativos de que o new rating tenha tido qualquer importância ou causado retornos anormais. E como possível justificativa para o resultado foi citado o método de avaliação do risco 
de crédito pelas agências classificadoras, em que os investidores conseguem saber do requerimento de avaliação de crédito de alguma forma.

Outra pesquisa realizada na Austrália foi desenvolvida por Chan, Edwards e Walter (2009), que utilizaram os retornos de longo prazo das ações para observar o período antes e depois da divulgação das alterações nos ratings. Os autores concluíram que existem retornos anormais que são estatisticamente significativos para os downgrades e os upgrades antes do anúncio da classificação de crédito. Porém, isso não foi observado para as janelas de evento de três dias para downgrades e upgrades e para a janela de evento de um ano para upgrades e possíveis upgrades, em que foram encontrados retornos normais após os anúncios.

Murcia, Murcia e Borba (2013) fizeram um estudo com o mesmo objetivo da presente pesquisa, em que analisaram o impacto dos downgrades, upgrades e ratings iniciais no preço de ações de empresas brasileiras, utilizando janelas de estimação. Esses autores utilizaram o Teste-T para testar as significâncias das três variáveis e encontraram retornos anormais positivos para upgrades e negativos para downgrades, porém, para os ratings iniciais os resultados foram mistos. Ao analisarem o retorno anormal médio cumulativo para cada janela do evento, os autores encontraram retornos anormais negativos estatisticamente significativos em quase todas as janelas realizadas para downgrades, isto é, verificaram que os retornos previstos foram maiores que os retornos observados. Já em relação aos upgrades e aos ratings iniciais, os resultados foram inconclusivos. Assim, concluíram que os ratings são de valor relevante, no entanto, essa relevância é maior quando eles abordam más notícias para o mercado, ou seja, quando há um downgrade.

Outra pesquisa que chegou à mesma conclusão de Murcia, Murcia e Borba (2013) foi o estudo realizado por Augusto (2015), que analisou como as mudanças nos ratings de crédito impactam o valor de mercado das empresas do Brasil. Os resultados indicaram que as divulgações dos ratings influenciam o preço das ações, porém esse efeito é mais enfatizado quando há downgrades. Além disso, Augusto (2015) concluiu que os investidores estão propensos a precipitar o anúncio do downgrade, já que o preço das ações diminui antes dessa data e volta a aumentar após ela.

Como estudo relacionado ao Watchlist, tem-se a pesquisa de Freitas e Minardi (2012), em que os autores analisaram se os preços das ações latino-americanas (Argentina, Brasil, Chile e México) são afetados por alterações nos rating ou por estarem na Watchlist (Credit Watch). Concluiu-se que os downgrades têm retornos negativos antes da data de mudança do rating e depois dessa data. Já para os upgrades, não houve indício de que os preços das ações são afetados de forma significativa por eles, mesmo que o Retorno Anormal Acumulado tenha sido positivo. Por último, os autores 
observaram que o efeito do Credit Watch para possíveis downgrades ou upgrades no preço das ações não foi significativo.

\section{METODOLOGIA}

\subsection{AMOSTRA E FONTE DOS DADOS}

A amostra utilizada neste estudo foi composta por 44 empresas brasileiras de capital aberto listadas na BM\&FBovespa que possuíam new rating e/ou mudanças de ratings (downgrades e upgrades) e/ou estavam na Watchlist durante os exercícios de 2000 a agosto de 2015. Conforme detalhado na Tabela 1, foram necessárias algumas exclusões para que se chegasse à amostra final preconizada no estudo.

Tabela 1 - Amostra total utilizada no estudo

\begin{tabular}{lr}
\hline Amostra total & 405 \\
\hline (+) Amostra inicial & 52 \\
(-) Empresas sem cotação diária & 18 \\
(-) Empresas com o rating retirado & 288 \\
(-) Empresas que não têm rating ou não têm o rating NRS LT emitidos pela Moody’s & 3 \\
(-) Empresas que não tiveram negociação em nenhum evento & 44 \\
(=) Total da amostra & \\
\hline Fonte: os autores.
\end{tabular}

Os ratings analisados foram os emitidos pela agência de classificação de risco Moody's, sendo considerados aqueles de longo prazo em escala nacional, já que se referem ao risco de crédito dentro de certo país, no caso, o Brasil. Em relação à quantidade de eventos considerados, estes encontram-se detalhados na Tabela 2.

Tabela 2 - Quantidade de eventos da amostra total

\begin{tabular}{lr}
\hline Evento & Total \\
\hline New Rating & 35 \\
Downgrade & 35 \\
Upgrade & 12 \\
On Watch - Possible Downgrade & 8 \\
On Watch - Possible Upgrade & 4 \\
Total & 94 \\
\hline Fonte: os autores.
\end{tabular}


Conforme evidenciado nas Tabelas 1 e 2, das empresas listadas na BM\&FBovespa, foram encontradas 44 companhias com ratings de longo prazo em escala nacional emitidos pela Moody's e um total de 94 eventos (new, downgrade, upgrade e ratings colocados em on watch). Além disso, oito das 44 empresas apresentaram eventos nos quais não havia cotação no dia da divulgação do rating ou não havia dados suficientes para calcular alguma das janelas, o que resultou na exclusão desses eventos. Porém, foram mantidos na amostra os demais eventos das companhias citadas que apresentaram cotações e dados suficientes para a inclusão no estudo.

\subsection{ESTUDO DE EVENTOS}

A utilização da metodologia do Estudo de Eventos se faz importante principalmente porque ela visa medir o impacto de uma informação conhecida de certa empresa, divulgada em algum momento, no desempenho dos preços e dos retornos dessa companhia nos mercados financeiros (LAMOUNIER; NOGUEIRA, 2005).

A primeira fase de um estudo de eventos, segundo MacKinlay (1997), é estabelecer o evento de interesse e definir o intervalo de tempo em que os preços das ações das empresas da base de dados serão analisados. Como esta pesquisa visa observar os efeitos dos ratings nos preços das ações, o evento é a divulgação dos ratings e a data do evento é a data do anúncio deles. Já a janela do evento é o período no qual os preços das ações das empresas serão examinados.

Assim como o estudo de Murcia, Murcia e Borba (2013), o objetivo com esta pesquisa não consistiu apenas em analisar o efeito da emissão de rating no preço das ações no dia da sua divulgação, por isso, a janela de eventos conteve alguns dias antes e após a emissão do rating para que fosse possível examinar o efeito antes, durante e após a divulgação. Assim, foi analisado o impacto dos anúncios de ratings nas seguintes janelas: 3 dias $(-1,+1), 7$ dias $(-3,3)$ e 11 dias $(-5,5)$, acrescentando que o dia zero é o dia em que o rating é emitido pela agência.

Após a definição da janela de eventos, foi determinada a janela de estimação que, de acordo com Rochman e Eid Junior (2006), é o período que tem a sua amostra como base para estimar o alfa e o beta do modelo relativo ao cálculo do retorno anormal. No caso deste estudo, a janela de estimação foi composta por 120 dias.

Por último, foi definida a janela de comparação, utilizando cinco dias após o evento. Segundo Fernandes (2013), nessa janela examina-se o comportamento da volatilidade do preço das ações e, assim, observa-se se ela retornará à normalidade. Na Figura 1 são resumidas as janelas anteriormente elencadas. 
Figura 1 - Janelas utilizadas para analisar o impacto dos anúncios de ratings da agência Moody’s

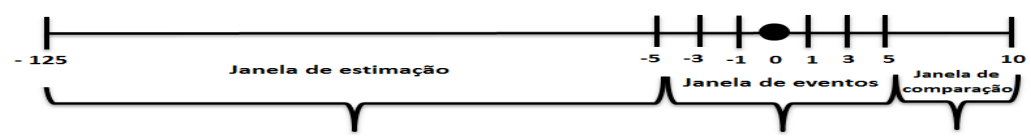

Fonte: os autores.

Para concluir o Estudo de Eventos, deve-se realizar outros procedimentos, como selecionar a amostra, medir o retorno anormal, definir o procedimento de estimação e o procedimento de teste, obter os resultados empíricos, interpretar os resultados e fazer conclusões a partir deles (CAMPBELL; LO; MACKINLAY, 1997), conforme já introduzido ao longo deste trabalho e detalhado nas subseções que seguem.

\subsubsection{Retornos normais}

Além do histórico das empresas que estão em Watchlist e do histórico dos ratings de longo prazo em escala nacional das companhias analisadas neste estudo, foi utilizado o histórico das cotações das ações dessas entidades, obtido no Economática ${ }^{\circledR}$ para o período de 2000 a 2015. A partir desses preços, foram calculados os retornos das ações, que servem de base para o cálculo dos retornos anormais, as variáveis de interesse deste estudo.

Soares, Rostagno e Soares (2002) afirmam que existem duas formas de se calcularem retornos das ações: a forma tradicional de cálculo (discreta) e a forma logarítmica (contínua). Porém, os autores ressaltam que a forma contínua é mais adequada quando se planeja fazer o uso de testes estatísticos paramétricos, em que se pede que seja utilizada a distribuição normal. A Equação 1 detalha tal forma de cálculo:

$$
R_{i t}=\ln \left(\frac{P_{i t}}{P_{i t-1}}\right)
$$

Em que: $\mathrm{R}_{\mathrm{it}}$ é o retorno da ação da empresa i no tempo t; $\mathrm{P}_{\text {it }}$ é o preço da ação da empresa i no tempo t; e $\mathrm{P}_{\text {it- } 1}$ é o preço da ação da empresa i no tempo $\mathrm{t}$ - 1 , ou seja, o preço do dia anterior. 


\subsubsection{Retornos anormais}

Segundo MacKinlay (1997), para calcular os retornos anormais podem ser usados modelos estatísticos e econômicos, como o Capital Asset Pricing Model (CAPM), o Arbitrage Pricing Theory (APT) e o modelo de retornos ajustados ao mercado. Além desses, outro modelo estatístico utilizado é o modelo de retornos ajustados ao risco e ao mercado (BROWN; WARNER, 1980). Este último, selecionado para a presente pesquisa, baseia-se no fato de que os retornos anormais das ações são dados pelos retornos individuais observados subtraídos dos retornos calculados por meio de um modelo de fator simples. Tal modelo de fator simples é calculado utilizando-se os dados da janela de estimação e uma regressão linear por meio de mínimos quadrados ordinários. A Equação 2 demonstra como os retornos anormais são calculados (ROCHMAN; EID JUNIOR, 2006):

$$
\mathrm{AR}_{\mathrm{it}}=\mathrm{R}_{\mathrm{it}}-\left(\alpha_{\mathrm{i}}+\beta_{\mathrm{i}} \mathrm{R}_{\mathrm{mt}}\right)
$$

Em que: $A R_{i t}$ é o retorno anormal da ação da empresa i no tempo t; $R_{i t}$ é o retorno da ação da empresa i no período t; $R_{m t}$ é o retorno do portfólio de mercado no período t; e $\alpha_{i}$ e $\beta_{i}$ são os parâmetros da regressão linear.

Conforme observado na Equação 2, o modelo de retorno ajustado ao mercado e ao risco para o cálculo dos retornos anormais apresenta, em sua fórmula, o retorno do portfólio de mercado, que, neste estudo, foi dado pelo Índice Bovespa (Ibovespa). Segundo a BM\&FBovespa (2015), a finalidade desse Índice é configurar-se como uma medida do desempenho médio das cotações dos ativos que refletem o mercado de ações brasileiro e dos que são comercializados.

\subsubsection{RETORNO ANORMAL MÉDIO E RETORNO ANORMAL MÉDIO ACUMULADO}

A partir dos retornos anormais, foi possível obter o cálculo da média desses retornos, ou seja, o Retorno Anormal Médio Average Abnormal Return (AAR) para todos os eventos n (ratings iniciais, downgrades, upgrades e Watchlist), fornecendo a média do retorno anormal para cada dia.

Ademais, a janela de evento abrange, geralmente, mais de um dia, sendo necessário definir algum critério de agregação dos retornos anormais. Isso pode ser realizado utilizando-se o Cumulative Abnormal Return (CAR). Nesse caso, o retorno 
anormal acumulado é calculado por meio da soma de todos os retornos anormais inclusos em uma janela de evento, de modo que será um evento individual de $t_{1}$ para $t_{2}$ , como evidenciado na Equação 3:

$$
\operatorname{CAR}_{i}\left(t_{1}, t_{2}\right)=\sum_{t_{1}}^{t_{2}} A R_{i t}
$$

Em que: $\mathrm{CAR}_{\mathrm{i}}$ é o retorno anormal acumulado da ação da empresa i entre o primeiro dia da janela do evento e o último dia da janela do evento. Porém, o que interessa para os resultados é o Retorno Anormal Médio Acumulado Cumulative Average Abnormal Returns (CAAR) entre quaisquer dois dias $t_{1}$ e $t_{2}$ dentro da janela do evento, calculado por meio da soma dos retornos anormais médios durante esse período.

\subsubsection{Teste-F e Teste-T}

Após o cálculo dos retornos anormais a partir do modelo de retornos ajustados ao risco e ao mercado, bem como da média destes, o primeiro teste a ser realizado é o Teste-F. Segundo Levine, Berenson e Stephan (2000), o Teste-F serve para observar, por meio de testes, a oscilação de duas amostras independentes, ou seja, se elas têm a mesma variância. Desse modo, é possível definir qual o pressuposto do Teste-T com Amostra Dupla é adequado para ser utilizado: se o Teste-T presumindo variações equivalentes ou presumindo variações diferentes.

Após a realização do Teste-F nos retornos anormais médios, é necessário verificar se esses retornos são significativos ou não, ou seja, testar as suas significâncias. Para tal, foi utilizado o Teste-T. Segundo Gujarati (2005), o teste de significância (Teste-T) utiliza os resultados da amostra para verificar se uma hipótese nula é falsa ou verdadeira, ou seja, rejeitar ou não rejeitar a hipótese nula $\left(H_{0}\right)$. Essa escolha entre aceitar ou rejeitar a hipótese nula é definida a partir do valor da estatística de teste obtida com as informações disponíveis. Como esse Teste se baseia em hipótese nula e alternativa $\left(H_{1}\right)$, foram criadas hipóteses para cada tipo de rating, de acordo com o estudo de Murcia, Murcia e Borba (2013):

a) New rating:

- $H_{0}$ : A divulgação de um new rating não provoca retornos anormais;

_ $H_{1}$ : A divulgação de um new rating provoca retornos anormais; 
b) Downgrades:

- $H_{0}$ : A divulgação de um downgrade não provoca retornos anormais negativos;

- $H_{1}$ : A divulgação de um downgrade provoca retornos anormais negativos;

c) Upgrades:

- $H_{0}$ : A divulgação de um upgrade não provoca retornos anormais positivos;

_ $H_{1}$ : A divulgação de um upgrade provoca retornos anormais positivos;

d) Watchlist:

- $H_{0}$ : A colocação de uma empresa em Watchlist não provoca retornos anormais positivos;

ـ $H_{1}$ : A colocação de uma empresa em Watchlist provoca retornos anormais positivos.

Ressalta-se que o Teste-F e o Teste-T foram realizados considerando os retornos anormais médios, calculados pelo modelo de retorno ajustado ao mercado e ao risco, para a janela de eventos $(-1 ;+1),(-3 ;+3)$ e $(-5 ;+5)$ e para os dias após a divulgação dos eventos $(0 ;+1),(0 ;+3)$ e $(0 ;+5)$, possibilitando análises e comparações acerca de suas significâncias.

\section{ANÁLISE DOS RESULTADOS}

\subsection{ANÁLISES DOS PARÂMETROS DA REGRESSÃO}

Para iniciar a análise dos resultados, o primeiro fator a ser observado é a significância nos parâmetros $\beta$ e $\alpha$ que foram previstos por meio de uma regressão, utilizando como base de cálculo os 120 dias da janela de estimação. Por meio da avaliação desses parâmetros, foi possível selecionar os eventos a serem testados mediante o Teste-F e o Teste-T.

Ao analisar-se o valor-p dos parâmetros $\hat{\beta}$ e $\hat{\alpha}$ da regressão estimada, foi utilizada a regra de decisão relacionada ao valor-p. Assim, foram retirados da amostra todos os $\hat{\beta}_{\mathrm{S}}$ cujos valores-p foram superiores a 0,05 . Em relação aos $\hat{\alpha}_{\mathrm{S}}$, como seus valores não foram grandes em termos absolutos, decidiu-se mantê-los no cálculo do retorno anormal, mesmo com valores-p superiores a 0,05, afetando os cálculos de maneira pouco significativa (TAKAMATSU; LAMOUNIER; COLAUTO, 2008). 
Diante do que foi tratado, do total de 94 eventos, foram encontrados 29 casos com valores $\hat{\beta}$ estatisticamente não significativos, ou seja, a amostra final passou a englobar 65 eventos.

\subsection{ANÁLISES DO TESTE-F PARA OS RETORNOS ANORMAIS MÉDIOS}

Após definir quais eventos apresentavam $\hat{\beta}$ s estatisticamente significativos, foi realizado o Teste-F para cada evento, analisando os três períodos da janela de estimação $(-1 ;+1),(-3 ;+3)$ e $(-5 ;+5)$ e três intervalos de tempo considerando os dias após a divulgação do evento $(0 ;+1),(0 ;+3),(0 ;+5)$. Nesta seção, detalham-se as janelas que apresentaram variância igual ou diferente, a partir dos resultados do Teste-F $(\mathrm{P}(\mathrm{F}<=\mathrm{f}))$.

Na Tabela 3 evidenciam-se os resultados do Teste supracitado.

Tabela 3 - Teste-F dos Retornos Anormais Médios para a janela de estimação

\begin{tabular}{rrrrrr}
\hline \multicolumn{7}{c}{$\mathbf{P}(\mathbf{F}<=\mathbf{f})$ unicaudal } \\
\hline Janela & New rating & Upgrade & Downgrade & On watch - possible downgrade & $\begin{array}{c}\text { On watch } \\
- \text { possible } \\
\text { upgrade }\end{array}$ \\
\hline$(-1 ;+1)$ & 0.1686 & 0.2369 & 0.1206 & 0.1867 & 0.1186 \\
$(-3 ;+3)$ & 0.1325 & 0.3717 & 0.1062 & $0.0406^{*}$ & 0.4228 \\
$(-5 ;+5)$ & $0.0331^{*}$ & 0.3698 & $0.0347^{*}$ & 0.3030 & 0.3453 \\
\hline
\end{tabular}

Fonte: os autores.

Nota: *Valores significativos ao nível de significância de 5\%.

A partir da análise da Tabela 3, conclui-se que apenas os eventos relativos a new rating e downgrade da janela $(-5 ;+5)$ e on watch - possible downgrade da janela $(-3 ;+3)$ rejeitaram a hipótese nula de variâncias equivalentes. Isso ocorreu para esses casos pois os valores-p do Teste-F foram inferiores a 0,05 , ou seja, foram estatisticamente significativos a um nível de significância de 5\%. Observa-se, desse modo, que apenas os três eventos citados apresentaram variâncias diferentes e, consequentemente, os outros, com valores-p superiores a 0,05, manifestaram variâncias equivalentes.

Na Tabela 4 detalham-se os resultados do Teste-F, considerando os dias após a divulgação do evento. 
Tabela 4 - Teste-F dos Retornos Anormais Médios para os dias após a divulgação do evento

\begin{tabular}{cccccc}
\hline & \multicolumn{1}{c}{$\mathbf{P}(\mathbf{F}<=\mathbf{f})$ unicaudal } \\
\hline Janela & New rating & Upgrade & $\begin{array}{c}\text { Downgra- } \\
\text { de }\end{array}$ & $\begin{array}{c}\text { On watch - possible } \\
\text { downgrade }\end{array}$ & $\begin{array}{c}\text { On watch - possible } \\
\text { upgrade }\end{array}$ \\
\hline$(0 ;+1)$ & 0.4350 & 0.4300 & 0.1911 & 0.4757 & 0.0891 \\
$(0 ;+3)$ & 0.2966 & 0.3015 & 0.2077 & 0.2645 & 0.2589 \\
$(0 ;+5)$ & 0.0837 & 0.2472 & 0.2099 & 0.0665 & 0.5208 \\
\hline
\end{tabular}

Fonte: os autores.

Nota: *Valores significativos ao nível de significância de 5\%.

De acordo com o que foi evidenciado na Tabela 4, nenhum dos eventos para as datas após a divulgação foi estatisticamente significativo ao nível de significância de 5\%, isto é, não houve eventos que apresentaram variâncias diferentes.

\subsection{ANÁLISES DO TESTE-T PARA OS RETORNOS ANORMAIS MÉDIOS}

Finalizados os resultados referentes ao Teste-F, realizou-se o Teste-T, que presumiu variâncias diferentes para as amostras que foram estatisticamente significativas e variâncias iguais para as amostras que não foram significativas nos resultados do Teste-F. O Teste-T foi realizado para os retornos anormais médios de cada evento, considerando os três períodos da janela de estimação $(-1 ;+1),(-3 ;+3)$ e $(-5 ;+5)$, conforme evidenciado na Tabela 5, e três intervalos de tempo para os dias após a divulgação do evento $(0 ;+1),(0 ;+3),(0 ;+5)$.

Tabela 5 - Teste-T dos Retornos Anormais Médios para a janela de estimação

\section{$\mathbf{P}(\mathbf{T}<=\mathbf{t})$ bicaudal}

\begin{tabular}{rrrrrr} 
Janela & New rating & Upgrade & Downgrade & $\begin{array}{c}\text { On watch-possible } \\
\text { downgrade }\end{array}$ & $\begin{array}{c}\text { On watch-possible } \\
\text { upgrade }\end{array}$ \\
\hline$(-1 ;+1)$ & 0.8012 & 0.0977 & 0.1083 & 0.0945 & 0.6381 \\
$(-3 ;+3)$ & 0.6429 & 0.2300 & 0.2228 & $0.0093^{*}$ & 0.8795 \\
$(-5 ;+5)$ & 0.3534 & 0.3965 & 0.0540 & 0.1489 & 0.7510 \\
\hline
\end{tabular}

Fonte: os autores.

Nota: *Valores significativos ao nível de significância de 5\%. 
A partir da análise da Tabela 5, percebe-se que somente o evento on watch - possible downgrade na janela $(-3 ;+3)$ mostrou-se estatisticamente significativo, indicando que, no período dessa janela, as ações foram afetadas de forma significativa pela divulgação desse evento. Em relação aos demais resultados, estes não foram considerados estatisticamente significativos (valor-p superior a 0,05), culminando no fato de que as ações não reagiram de forma significativa à publicação desses eventos.

Na Tabela 6, apresentam-se os resultados do Teste-T, considerando os dias após a divulgação do evento.

Tabela 6 - Teste-T dos Retornos Anormais Médios para os dias após a divulgação do evento $\mathbf{P}(\mathbf{T}<=\mathbf{t})$ bicaudal

\begin{tabular}{crrrrr} 
Janela & New rating & Upgrade & Downgrade & $\begin{array}{c}\text { On watch-possible } \\
\text { downgrade }\end{array}$ & $\begin{array}{c}\text { On watch - possib- } \\
\text { le upgrade }\end{array}$ \\
\hline$(0 ;+1)$ & 0.9238 & 0.6514 & 0.1172 & 0.1579 & 0.2913 \\
$(0 ;+3)$ & 0.7824 & 0.6791 & 0.1415 & 0.2616 & 0.6536 \\
$(0 ;+5)$ & 0.6627 & 0.5895 & 0.2401 & 0.6052 & 0.4462 \\
\hline
\end{tabular}

Fonte: os autores.

Nota: *Valores significativos ao nível de significância de 5\%.

Os eventos elencados na Tabela 6 não apresentaram significância nos resultados relacionados ao Teste-T. Isso evidencia que, para períodos após os eventos, especificados na Tabela em análise, os preços das ações não sofreram alterações mediante a divulgação de new ratings, upgrades, downgrades, on watch - possible downgrade e on watch - possible upgrade.

\subsection{ANÁLISES DOS GRÁFICOS PARA OS RETORNOS ANORMAIS MÉDIOS}

Os gráficos delineados nesta seção apresentam os retornos anormais médios dos eventos relativos aos new ratings, downgrades, upgrades e on watch - possible downgrades e on watch - possible upgrades durante os períodos da janela de eventos e da janela de comparação. Além disso, a respeito da janela de comparação $(+5 ;+10)$, não é possível afirmar que os retornos retornaram à normalidade, visto que nenhum dos eventos apresentou retornos anormais significativos pelo Teste-T. Portanto, como os preços das ações não reagiram aos eventos, os retornos não apresentaram, em mo- 
mento algum, um comportamento de normalidade. No Gráfico 1 apresenta-se o retorno anormal médio relativo ao new rating, enquanto no Gráfico 2 se evidencia o retorno anormal médio relativo ao downgrade.

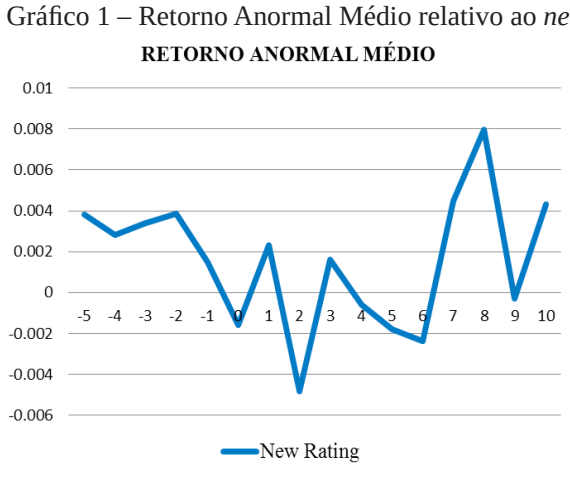

Fonte: os autores.
Gráfico 2 - Retorno Anormal Médio relativo ao downgrade

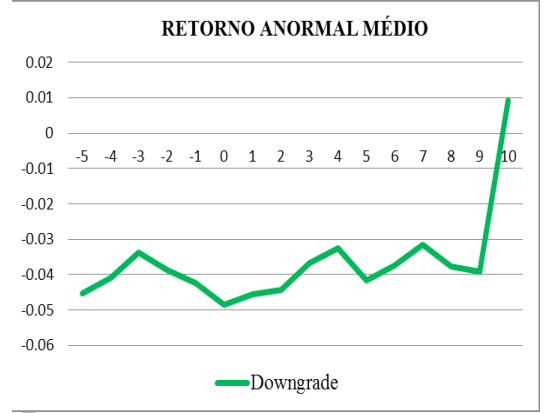

Fonte: os autores.

Ao analisar-se o Gráfico 1, verifica-se que, no decorrer do período, houve retornos anormais e, em sua grande parte, positivos, ou seja, em sua maioria os retornos observados foram maiores do que os previstos. Porém, a realização do Teste-T demonstrou que não houve retornos anormais significativos relacionados aos new ratings, o que descarta a hipótese de que os preços das ações reagem aos eventos relativos a eles.

Caso o Teste-T para os retornos anormais relativos aos downgrades tivesse apresentado resultado significativo, seria possível inferir, mediante a análise do Gráfico 2, que os preços das ações reagiriam de maneira negativa. Isso quer dizer que, caso os retornos anormais tivessem se mostrado estaticamente significativos, o retorno previsto teria sido maior do que o retorno observado, sugerindo que os preços das ações caem em decorrência de downgrades.

No que se refere ao upgrade, os resultados encontram-se detalhados no Gráfico 3. A mesma ocorrência foi investigada em relação ao on watch - possible downgrade, conforme evidenciado no Gráfico 4. 
Gráfico 3 - Retorno Anomal Médio relativo ao upgrade RETORNO ANORMAL MÉDIO

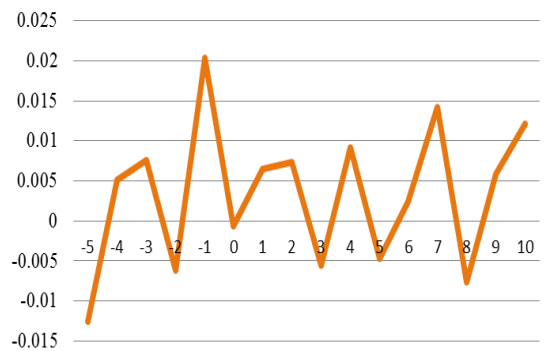

Fonte: os autores.
Gráfico 4 - Retorno Anormal Médio relativo ao on watch - possible downgrade

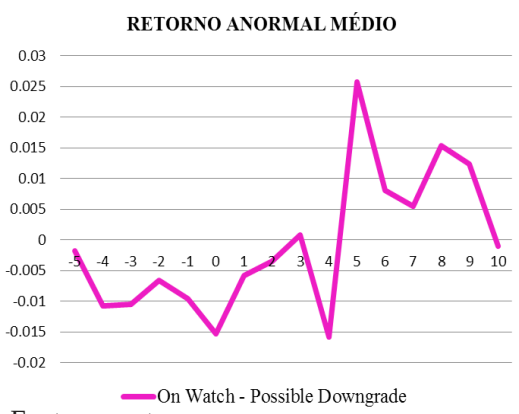

Fonte: os autores.

Situação similar à verificada no que se refere aos retornos anormais relativos ao new rating ocorreu com os concernentes a upgrade, que, em sua maioria, foram positivos, sugerindo uma reação positiva do mercado quanto aos preços das ações (Gráfico 3). Entretanto, os Retornos Anormais Médios não foram estatisticamente significativos segundo o Teste-T, o que denota que as cotações não sofrem impacto com o anúncio de upgrades.

Em relação aos retornos anormais apresentados no Gráfico 4, há indícios de que existem retornos anormais negativos na maior parte do período, sugerindo que o mercado visualiza o evento on watch - possible downgrade como uma má notícia. E, nos últimos cinco dias, observam-se retornos anormais positivos, indicando que após um período da divulgação do evento os retornos foram relativamente superiores às expectativas. Salienta-se, no entanto, que como o Teste-T não foi significativo para o evento citado, as afirmações anteriores não se confirmam.

Por fim, no Gráfico 5, apresenta-se o Retorno Anormal Médio relativo ao on watch - possible upgrade.

Gráfico 5 - Retorno Anormal Médio relativo ao on watch - possible upgrade

RETORNO ANORMAL MÉDIO

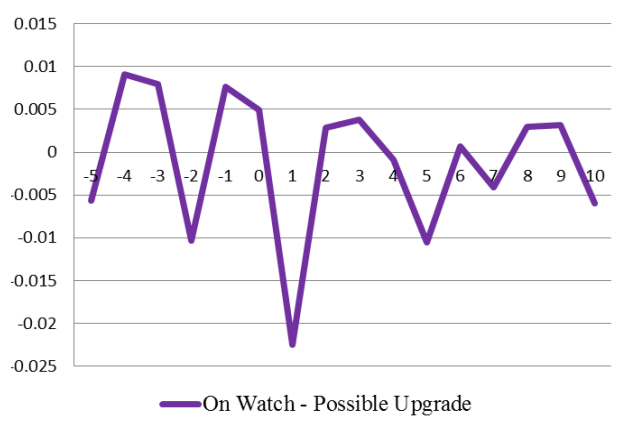

Fonte: os autores. 
A partir da análise do Gráfico 5, observa-se que os retornos anormais não apresentaram tendência aparente, variando entre negativos e positivos durante todos os períodos da janela de estimação. Verifica-se que perto do dia zero até o dia um, há uma queda considerável do retorno anormal, indicando que os preços das ações poderiam reagir a uma divulgação de on watch - possible upgrade. No entanto, essa verificação não se concretiza quando se observam os resultados do Teste-T.

Após o exame dos gráficos relativos aos retornos anormais médios para cada um dos eventos estudados, optou-se por realizar análise similar considerando os retornos anormais médios acumulados. No Gráfico 6 apresenta-se o Retorno Anormal Médio acumulado para o evento new rating.

Gráfico 6 - Retorno Anormal Médio Acumulado relativo ao new rating

\section{RETORNO ANORMAL MÉDIO ACUMULADO}

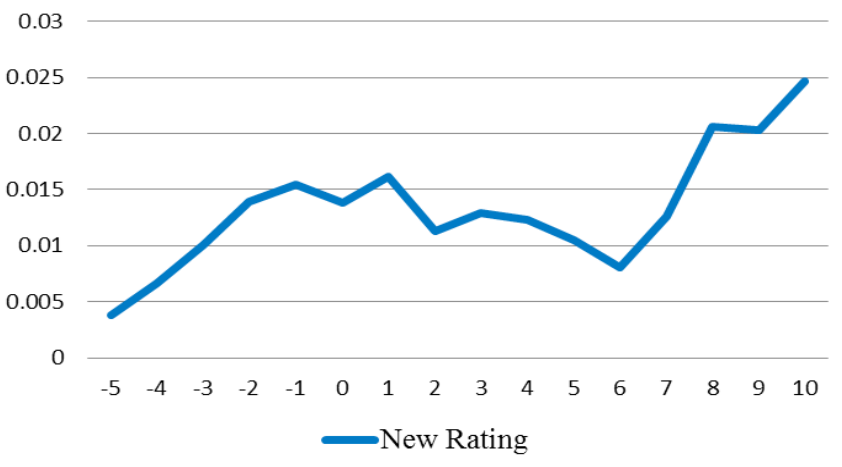

Fonte: os autores.

Observa-se que o Retorno Anormal Médio acumulado relativo ao new rating é sempre positivo ao longo do tempo, o que demonstra que, caso o Teste-T para o new rating tivesse sido significativo, o anúncio desse evento seria visto como uma boa notícia para o mercado.

Análise similar foi realizada quanto ao downgrade, conforme detalhado no Gráfico 7. 
Gráfico 7 - Retorno Anormal Médio acumulado relativo ao downgrade

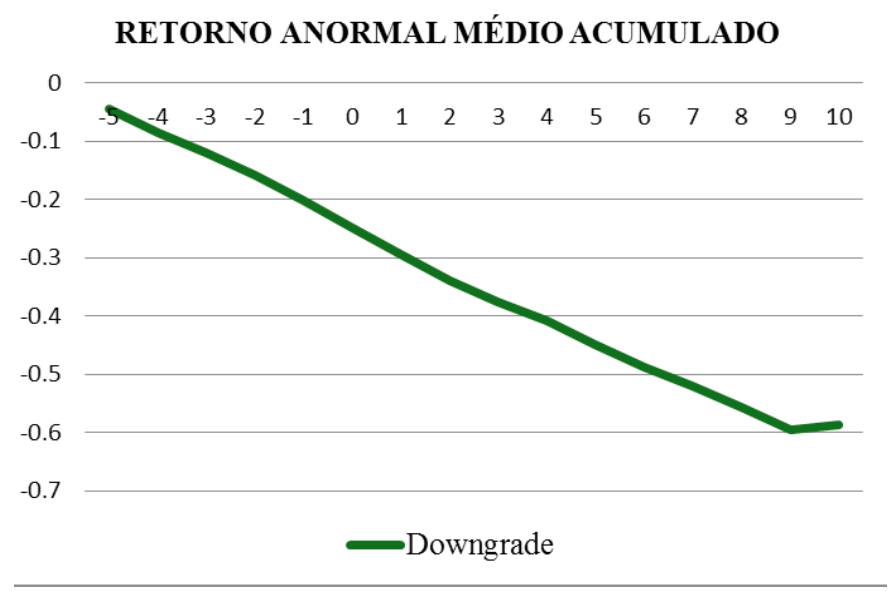

Fonte: os autores.

A análise do Gráfico 7 permite inferir que em todos os períodos os retornos anormais tendem a continuar negativos para downgrades, coincidindo com uma série de estudos que concluíram uma reação negativa do mercado para esse evento. Ao acumular os retornos, observa-se, mediante a análise gráfica, que após o anúncio do downgrade estes se tornam cada vez mais negativos, porém, isso não se confirma a partir dos resultados do Teste-T.

No Gráfico 8 evidencia-se o Retorno Anormal Médio acumulado relativo ao upgrade.

Gráfico 8 - Retorno Anormal Médio acumulado relativo ao upgrade

\section{RETORNO ANORMAL MÉDIO ACUMULADO}

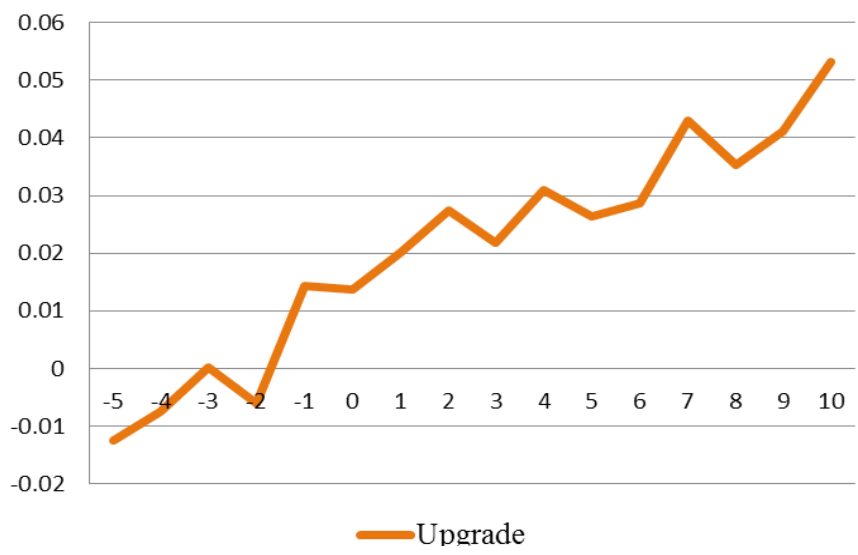

Fonte: os autores. 
No início do Gráfico 8 verifica-se que alguns dias antes da data de divulgação do upgrade os retornos tendem a se acumular negativamente, demonstrando que talvez o mercado não esteja prevendo um anúncio de upgrade. Em seguida, os retornos acumulados se tornam cada vez mais positivos, manifestando uma boa reação do mercado em relação ao anúncio do evento, na hipótese de que os retornos anormais fossem significativos.

Quanto ao evento on watch - possible downgrade, a análise pode ser realizada observando-se o Gráfico 9.

Gráfico 9 - Retorno Anormal Médio acumulado relativo ao on watch - possible downgrade

RETORNO ANORMAL MÉDIO ACUMULADO

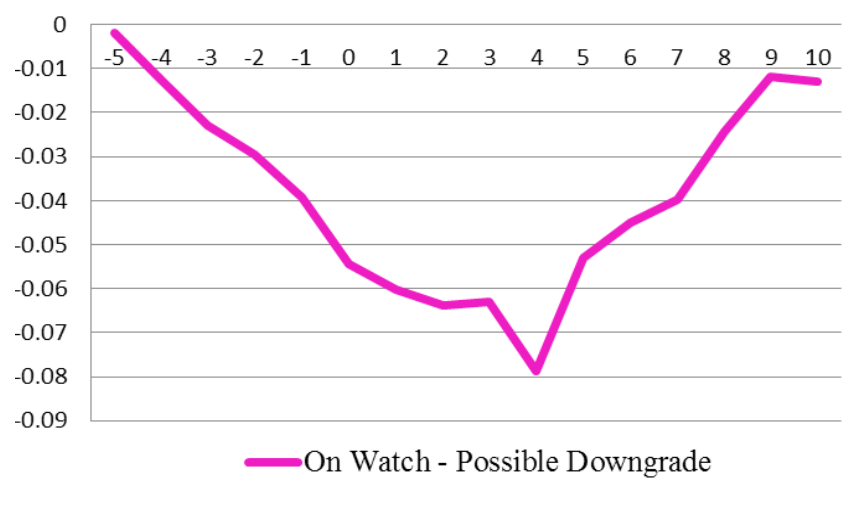

Fonte: os autores.

No que se refere ao evento em questão, caso o resultado do Teste-T tivesse sido estaticamente significativo, o mercado mostraria uma reação negativa em relação a esse evento durante todo o período, ou seja, o anúncio de um on watch - possible downgrade seria visto como uma má notícia pelo mercado, refletindo no preço das ações.

No Gráfico 10, finalizando a análise, apresenta-se o retorno anormal médio acumulado relativo ao on watch - possible upgrade. 
Gráfico 10 - Retorno Anormal Médio acumulado relativo ao on watch - possible upgrade

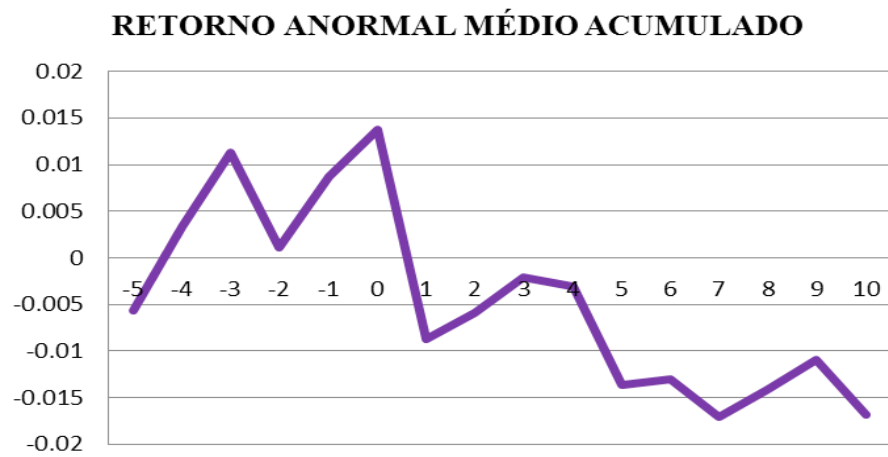

On Watch - Possible Upgrade

Fonte: os autores.

Ao contrário do que foi observado para os retornos anormais médios acumulados relativos aos upgrades, o Gráfico 10 evidencia que, antes do anúncio do evento, os retornos estavam positivos, porém, após a divulgação se tornaram cada vez mais negativos. Tal situação demonstra que o efeito negativo sobre o preço das ações se sobrepõe ao positivo após o anúncio do evento. No entanto, conforme já observado nas análises anteriores, como o Teste-T não foi significativo para esse evento, não foi possível confirmar o fato descrito.

\subsection{COMPARAÇÃO COM OUTRAS PESQUISAS}

Na presente pesquisa, os eventos relacionados a new ratings não foram considerados significativos, indicando que os preços das ações não reagem a esse tipo de evento. Esse resultado coincidiu com o encontrado por Callado et al. (2008), que, utilizando um estudo de eventos relacionado a instituições financeiras negociadas na BM\&FBovespa, não obtiveram evidências significativas de que a emissão de um new rating causa retornos anormais.

Freitas e Minardi (2012), por sua vez, utilizando o retorno anormal acumulado, observaram se os preços das ações da Argentina, Brasil, Chile e México são afetados por alterações nos rating ou por estarem na Watchlist. Um dos resultados desse estudo que foi corroborado na presente pesquisa é o relacionado aos upgrades, em que não foi encontrado indício de que os preços das ações são afetados de forma significativa por eles, mesmo que o Retorno Anormal acumulado tenha sido positivo. 
Quanto à Watchlist, não foram encontrados resultados significativos, ou seja, infere-se que eventos relacionados à Watchlist (para possíveis downgrades ou upgrades) não afetam as cotações. Ainda utilizando o estudo de Freitas e Minardi (2012), os autores não encontraram um efeito tão significativo para Credit Watch quanto para os downgrades. Na pesquisa, esse acontecimento foi justificado em relação aos países de onde a amostra foi retirada, pois os seus investidores são propensos a aguardarem a alteração do rating, fazendo com que o Credit Watch não seja demasiado relevante.

Os resultados do presente estudo demonstraram, ainda, que os downgrades não afetam de forma significativa os preços das ações, de forma similar ao verificado no estudo de Abad-Romero e Robles-Fernandez (2006). Estes últimos utilizaram ratings emitidos pela Moody’s, Standard \& Poor e Fitch IBCA no mercado da Espanha e observaram o impacto das alterações dos ratings dos títulos das companhias nos retornos de mercado e no risco sistemático. Concluíram que os downgrades estão associados a uma estabilidade no risco da companhia, pois as agências classificadoras de risco de crédito publicam um grau maior absoluto de risco, mas o mercado capta um risco sistemático menor.

\section{CONSIDERAÇÕES FINAIS}

O objetivo principal com esta pesquisa foi analisar o impacto das publicações de new ratings, da Watchlist e das variações dos ratings de crédito (downgrades e upgrades) emitidos pela Moody's, nos retornos das ações de 44 empresas de capital aberto listadas na BM\&FBovespa.

Considerando a teoria de eficiência de mercado, esperava-se que o mercado reagisse positivamente às boas notícias (upgrades) e negativamente às más notícias (downgrades). Porém, ao utilizar o estudo de eventos e o Teste-T nos retornos anormais médios dos anúncios para as janelas $(-1 ;+1),(-3 ;+3),(-5 ;+5)$ e para os dias após as publicações $(0 ;+1),(0 ;+3),(0 ;+5)$, verificou-se que os resultados não foram consistentes com a teoria de eficiência de mercado, mostrando-se não significativos. Inferiu-se, a partir do teste, que new ratings, upgrades, downgrades, on watch - possible upgrade e on watch - possible upgrade não afetam os retornos das ações de maneira significativa em nenhuma janela de eventos analisada neste estudo.

Tal resultado pode ser explicado pelo fato de que foram analisadas apenas 44 empresas e somente os dados da agência classificadora Moody’s. Ressalta-se que outros estudos que utilizaram uma amostra maior e mais de uma agência encontraram resultados significativos para alguns dos eventos. Desse modo, verificou-se que 
os ratings nacionais de longo prazo relativos a downgrades, upgrades, new ratings, on watch (para possíveis downgrades e upgrades) não trazem novas informações ao mercado, fazendo com que este não reaja aos eventos, além de não apresentarem valor preditivo sobre as futuras cotações.

Como recomendação para estudos futuros, destaca-se a possibilidade de utilização de dados de mais de uma agência classificadora de risco além da Moody’s, de forma a obter uma quantidade maior de eventos para análise. Uma segunda sugestão seria comparar os resultados entre as agências, ou seja, verificar se podem ser encontrados resultados similares realizando-se a mesma pesquisa com dados de agências distintas. Por fim, recomenda-se analisar como fatores que explicitam características das empresas da amostra, como tamanho, alavancagem e mudança de ratings, poderiam impactar os retornos anormais cumulativos.

\section{REFERÊNCIAS}

ABAD-ROMERO, P.; ROBLES-FERNANDEZ, M. D. Risk and return around bond rating changes: New evidence from the Spanish stock market. Journal of Business Finance \& Accounting, v. 33, i. 5, p. 885-908, 2006.

AUGUSTO, D. D. N. Impacto das classificações de rating no valor das empresas brasileiras. 2015. 56 p. Dissertação (Mestrado em Economia)-Fundação Getúlio Vargas, São Paulo, 2015.

BELO, N. M.; BRASIL, H. G. Assimetria informacional e eficiência semiforte do mercado. Revista de Administração de Empresas, Minas Gerais, v. 46, p. 48-57, 2006. Edição Especial.

BM\&FBOVESPA. Índice Bovespa - Ibovespa. Disponível em: $<$ http://www.bmfbovespa.com.br/indices/ResumoIndice.aspx?Indice=IBOVESPA\&idioma=pt-br> . Acesso em: 19 abr. 2015.

BM\&FBOVESPA. Rating. Disponível em: <http://www.bmfbovespa.com.br/ shared/iframe.aspx?altura=600\&idioma=pt-br\&url=http://www.bmfbovespa.com.br/ rendafixa/FormConsultaDebRatingEmissoes.asp>. Acesso em: 19 abr. 2015.

BROWN, S.; WARNER, J. Measuring security price performance. Journal of Financial Economics, v. 8, i. 3, 1980.

CALDERONI, F.; COLLA, P.; GATTI, S. Ratings changes: The European evidence. CAREFIN Research Paper Series, 2009. 
CALLADO, A. A. C. et al. O impacto da primeira emissão de conceito de risco crédito sobre os preços das ações: Um estudo empírico sobre a reação do mercado acionário brasileiro para o setor bancário. Revista Ciências da Administração, v. 14, n. 1, p. 80-88, 2008.

CAMPBELL, J. Y.; LO, A. W.; MACKINLAY, A. C. The Econometrics of Financial Markets. Princeton: Princeton University Press, 1997.

CHAN, P. T.; EDWARDS, V.; WALTER, T. The information content of Australian credit ratings: A comparison between subscription and non-subscription-based credit rating agencies. Economic Systems, v. 33, n. 1, p. 22-44, 2009.

CREIGHTON, A.; GOWER, L.; RICHARDS, A. J. The impact of rating changes in Australian financial markets. Pacific - Basin Finance Journal, v. 15, i. 1, p. 1-17, 2007.

DAMASCENO, D. L.; ARTES, R.; MINARDI, A. M. A. F. Determinação de rating de crédito de empresas brasileiras com a utilização de índices contábeis. Revista de Administração, São Paulo, v. 43, n. 4, 2008.

FAMA, E. F. Efficient capital markets: a review of theory and empirical work. The Journal of Finance, v. 25, i. 2, p. 421-423, maio 1970.

FERNANDES, S. M. Os efeitos do disclosure ambiental negativo involuntário: um estudo de evento nas companhias brasileiras com alto índice de poluição. Revista de Contabilidade e Organizações, São Paulo: FEA/USP, v. 7, n. 17, p, 59-72, jan./abr. 2013.

FREITAS, A. P. M.; MINARDI, A. M. A. F. The impact of credit rating changes in Latin American stock markets. In: BALAS ANNUAL CONFERENCE, 1., 2012, Rio de Janeiro. Anais... Rio de Janeiro: Balas Annual Conference, 2012.

GUJARATI, D. N. Econometria Básica. 3. ed. São Paulo: Pearson Education do Brasil, 2005.

HILL, C. A. Regulating the Rating Agencies. Washington University Law Quarterly, v. 82, 2004.

JENSEN, M. C. Some anomalous evidence regarding market efficiency. Journal of Financial Economics, Amsterdam: North Holland, v. 6, i. 1, p. 95-101, Mar. 1978. 
LAMOUNIER, W. M.; NOGUEIRA, E. M. Estudo de eventos: procedimentos e estudos empíricos. In: SEMINÁRIO DE GESTÃO DE NEGÓCIOS, 2., 2005, Curitiba. Anais... Curitiba: Seminário de Gestão de Negócios, 2005. 1 CD-ROM.

LEVINE, D. M.; BERENSON, M. L.; STEPHAN, D. Estatística: teoria e aplicações usando microsoft excel em português. Rio de Janeiro: LTC, 2000.

LI, H.; VISALTANACHOTI, N.; KESAYAN, P. The effects of credit rating announcements on shares in the Swedish stock market. International Journal of Finance, v. 16, i. 1, p. 2872-2896, 2003.

MACKINLAY, A. C. Event Studies in Economics and Finance. Journal of Economic Literature, v. 35, i. 1, p. 13-39, Mar. 1997.

MALUF FILHO, J. A. Eficiência do mercado de opções da Bolsa de Valores de São Paulo. Revista de Administração, São Paulo: FEA/USP, v. 26, n. 3, p. 12- 22, jul./ set. 1991.

MOODY'S. Sistema de rating da Moody’s. Disponível em: <https://www.moodys. com/sites/products/ProductAttachments/sistemaderatingmoodys.pdf > . Acesso em: 19 abr. 2015.

MURCIA, F. C. S.; MURCIA, F. D. R.; BORBA, J. A. The Informational Content of Credit Ratings in Brazil: An Event Study. Rev. Bras. Finanças, Rio de Janeiro, v. 11, n. 4, p. 503-526, dez. 2013.

MURCIA, F. C. S. Rating de crédito no Brasil: Fatores Determinantes e Impacto de Anúncios nos Preços das Ações. 2013. 155 p. Tese (Doutorado em Administração)-Universidade Federal de Santa Catarina, Florianópolis, 2013.

PAPAIKONOMOU, V. Credit rating agencies and global financial crisis. Studies in Economics and Finance, v. 27, i. 2, p. 161-174, 2010.

ROCHMAN, R. R.; EID JUNIOR, W. E. Insiders conseguem retornos anormais? Estudos de eventos sobre as operações de insiders das empresas de governança corporativa diferenciada na Bovespa. In: ENCONTRO ANUAL DA ANPAD, 30., 2006, Salvador. Anais... Salvador: Anpad, 2006. 1 CD-ROM.

\section{SALES, B. F. Desenvolvimento de metodologia de rating baseado no modelo} ordered probit. 2006. 44 p. Dissertação (Mestrado em Finanças e Economia Empresarial)-Fundação Getúlio Vargas, Rio de Janeiro, 2006. 
SIH, A. Predição do grau de ratings corporativos. 2006. 84 p. Dissertação (Mestrado em Engenharia Elétrica)-Pontifícia Universidade Católica do Rio de Janeiro, Rio de Janeiro, 2006.

SOARES, R. O.; ROSTAGNO, L. M.; SOARES, K. T. C. Estudo de Evento: O Método e as Formas de Cálculo Do Retorno Anormal. In: ENCONTRO ANUAL DA ANPAD, 26., 2002, Salvador. Anais... Salvador: Anpad, 2002. 1 CD-ROM.

TAIB, H. M. et al. Do announcements of corporate bond rating revisions matter? Oct. 12, 2009.

TAKAMATSU, R. T.; LAMOUNIER, W. M.; COLAUTO, R. D. Impactos da divulgação de prejuízos nos retornos de ações de companhias participantes do Ibovespa. Revista Universo Contábil, Blumenau, v. 4, n. 1, p. 46-63, jan./mar. 2008.

\section{Como citar este artigo:}

\section{ABNT}

SILVEIRA, Rafaela Augusta Cunha; TAKAMATSU, Renata Turola; AVELINO, Bruna Camargos. Impacto dos ratings de crédito nas ações de empresas de capital aberto no Brasil. RACE, Revista de Administração, Contabilidade e Economia, Joaçaba: Ed. Unoesc, v. 16, n. 2, p. 573-602, maio/ago. 2017. Disponível em: $<$ http://editora.unoesc.edu.br/index.php/race>. Acesso em: dia/mês/ano.

APA

Silveira, R. A. C., Takamatsu, R. T., \& Avelino, B. C. (2017). Impacto dos ratings de crédito nas ações de empresas de capital aberto no Brasil. RACE, Revista de Administração, Contabilidade e Economia, 16(2), 573-602. Recuperado em dia/mês/ano, de http://editora.unoesc.edu.br/index.php/race 
